\title{
An Equilibrium Study on Trichloroethylene Adsorption and Desorption from ZSM-5 in Water
}

\author{
Harmonie A. Hawley ${ }^{1,2, *}$, John Bergendahl ${ }^{2}$, Robert Thompson ${ }^{3}$ \\ ${ }^{1}$ The University of Texs at Tyler, Department of Civil Engineering, 3900 University Blvd, Tyler, TX 75799, USA \\ ${ }^{2}$ Worcester Polytechnic Institute, Department of Civil \& Environmental Engineering, 100 Institute Rd, Worcester, MA 01609, USA \\ ${ }^{3}$ Worcester Polytechnic Institute, Department of Chemical Engineering, 100 Institute Rd, Worcester, MA 01609, USA \\ *Corresponding Author: hhawley@fullerton.edu
}

Copyright (C) 2013 Horizon Research Publishing All rights reserved.

\begin{abstract}
Zeolite ZSM-5 was found to effectively remove trichloroethylene (TCE) from water.This study measured the extent of uptake of TCE from water, determined adsorption isotherms, and evaluated desorption of TCE from ZSM-5.TCE was rapidly removed from water and followed linear and Freundlich adsorption isotherms.Desorption of TCE from ZSM-5 occurred, but the linear isotherm was still an acceptable model for the removal of TCE from water with ZSM-5.
\end{abstract}

Keywords Adsorption, Zeolites, Trichloroethylene Isotherms

\section{Introduction}

Zeolites have been found to remove organic contaminants from water by allowing organic molecules smaller than the zeolite pore size to enter the zeolite pore space and adsorb to internal surfaces.Zeolites are referred to as molecular sieves due to their molecular size exclusion properties.ZSM-5 is characterized by a relatively small pore size and high $\mathrm{SiO}_{2}$ to $\mathrm{Al}_{2} \mathrm{O}_{3}$ ratio of approximately 1,000 [1].ZSM-5 is a hydrophobic zeolite and shown to remove hydrocarbons from water $[1,2,3,4]$.Previous studies have shown that the zeolite ZSM-5 is more efficient than activated carbon for removing trichloroethylene (TCE) from water $[1,5,6,7]$.

TCE $\left(\mathrm{C}_{2} \mathrm{HCl}_{3}\right)$ is one of the most common groundwater contaminants in the United States (US).The US Environmental Protection Agency (EPA) set a maximum drinking water limit of $5 \mu \mathrm{g} / \mathrm{L}$ TCE due to its potential as a health hazard.The typical levels reported in water are below $200 \mu \mathrm{g} / \mathrm{L}[8,9]$.Thus, the sorption experiments in this study were conducted with an intitial TCE concentration of 500 $\mu \mathrm{g} / \mathrm{L}$. This concentration was chosen as an environmentally relevant concentration.

In practice the concentration of TCE in a water stream is not constant but varies over time, thus desorption may play a role in the equilibrium conditions. This study first identified the adsorption of TCE and then presented a modified isotherm for TCE adsorption to ZSM-5 that takes into account desorption due to reduced TCE concentrations in the bulk solution.

\section{Materials and Methods}

TCE was purchased as ACS Grade with an assay of $99.9 \%$ from Fisher Scientific (Fairlawn, NJ).All water used in experiments was purified on site with a Barnstead/Thermolyne E-pure water purification system (Dubuque, Iowa).The zeolite ZSM-5 was obtained from Grace-Davidson (Columbia, MD).TCE was quantified using Solid Phase Microextraction (Supelco, Bellefonte, PA) combined with an Agilent Series 6890 Gas Chromatograph equipped with a Flame Ionization Detector (GC).A fused silica column with a length of $30 \mathrm{~m}$, inner-diameter of 0.32 $\mathrm{mm}$, and a film thickness of $0.25 \mu \mathrm{m}$ was purchased from J\& W Scientific (Folsom, CA).

Solid Phase Microextraction (SPME) was used to extract and concentrate aqueous TCE for GC analysis [10].The SPME fiber used was a silica fiber coated with $85 \mu \mathrm{m}$ of Carboxen/polydimethylsiloxane.New fibers were conditioned at $260^{\circ} \mathrm{C}$ for one hour in the $\mathrm{GC}$ inlet, followed by contacting purified water until the fiber was stable.The SPME fiber was left in aqueous solution to equilibrate for 25 minutes, and then placed into the GC inlet for ten minutes to ensure that all organics were removed from the fiber.The inlet temperature was set to $240{ }^{\circ} \mathrm{C}$.The detection limit was 1 $\mu \mathrm{g} / \mathrm{L}$ TCE.

\subsection{Adsorption}

Adsorption was determined by adding $0.1 \mathrm{~g}$ of zeolite to a $43 \mathrm{~mL}$ glass vial, which was then filled with a $500 \mu \mathrm{g} / \mathrm{L} \mathrm{TCE}$ solution. The vial was wrapped in aluminum foil to prevent TCE degradation by photolysis. The vials were placed on their sides on a shaker table set to $125 \mathrm{rpm}$. Two vials were sacrificed at each time increment: $0,15,20,30,60,90,120$, 
and 180 minutes.Vials were centrifuged at 3,000 rpm for ten minutes; then $21.5 \mathrm{~mL}$ of supernatant was removed from each vial and transferred to one clean, $43 \mathrm{~mL}$ glass vial.The supernatant was analyzed for TCE concentration using SPME and GC.The temperature of the solution was recorded at $23{ }^{\circ} \mathrm{C}$ and maintained constant for all of the experiments.

\subsection{Adsorption Equilibrium}

Adsorption equilibrium was investigated by contacting different concentrations of TCE solution with $0.1 \mathrm{~g}$ of ZSM-5.The vials were left to equilibrate for 24 hours on a shaker table.After 24 hours, the vials were centrifuged and the supernatant transferred to clean vials. The TCE remaining in solution was quantified via SPME and GC.The concentrations of TCE added to the vials were 500; 1,000; 1,$250 ; 1,500 ; 2,500 ; 3,000 ; 12,000$; and $20,000 \mu \mathrm{g} / \mathrm{L}$.To create an equilibrium isotherm, the mass of TCE adsorbed to the zeolite per mass of zeolite $\left(\mathrm{q}_{\mathrm{e}}\right)$ was plotted against the equilibrium aqueous concentration of TCE $\left(\mathrm{C}_{\mathrm{e}}\right)$. The mass sorbed to the zeolite was the original mass $\left(\mathrm{M}_{\mathrm{o}}\right)$ added to the vial minus the mass of TCE remaining in the supernatant $\left(\mathrm{M}_{\mathrm{e}}\right)$ [11].

\section{Results and Discussion}

Each test was conducted in triplicate; for all Figures the plotted values are the average of each test and the error bars are the $95 \%$ confidence intervals.

\subsection{Adsorption}

ZSM-5 rapidly removed TCE from the water as shown in Figure 1.The starting concentration was $500 \mu \mathrm{g} / \mathrm{L}$ and after 20 minutes the concentration in the water was less than 50 $\mu \mathrm{g} / \mathrm{L}$.After 180 minutes, approximately $95 \%$ was removed from water.An adsorption rate constant was determined by plotting $\ln \left[\mathrm{C}_{\mathrm{t}} / \mathrm{C}_{\mathrm{o}}\right]$ versus time. The slope of the line is the adsorption rate constant and was found to be $0.0105 \mathrm{~min}^{-1}$.At this time the authors are unable to find other research related to the rate of adsorption of TCE by ZSM-5.

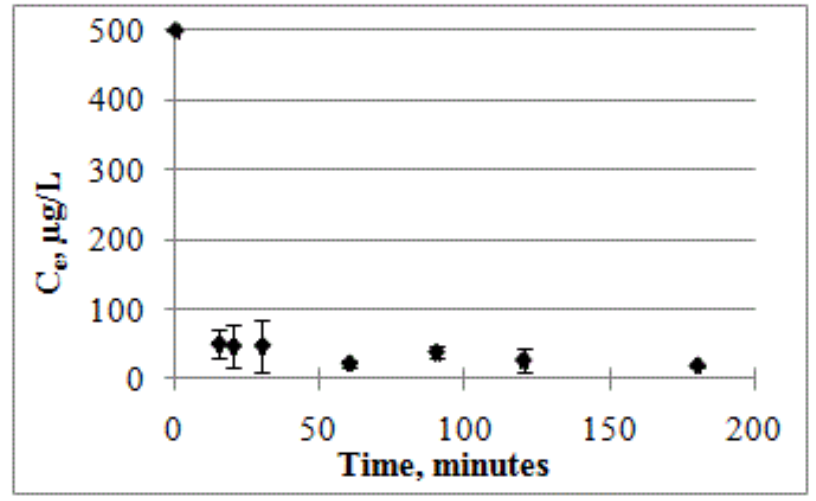

Figure 1. Sorption kinetics of TCE to $0.1 \mathrm{~g}$ of ZSM-5

\subsection{Adsorption Isotherms}

Three isotherms were investigated to model TCE sorption to ZSM-5: linear, Freundlich, and Langmuir.The linear isotherm is shown in Figures 2 and 3.The correlation was quite high for the linear isotherm $\left(\mathrm{R}^{2}=0.9966\right)$. Figure 3 is an enlarged view of the lower concentration equilibrium points. The correlation was slightly lower (0.9802), but still acceptable.The data were linearized to fit to the other two models. The Freundlich isotherm is represented by (1), where $\mathrm{K}$ and $\mathrm{n}$ are characteristic constants which represent the adsorptive capacity and the adsorption intensity, respectively, of the adsorbent material [11,12].

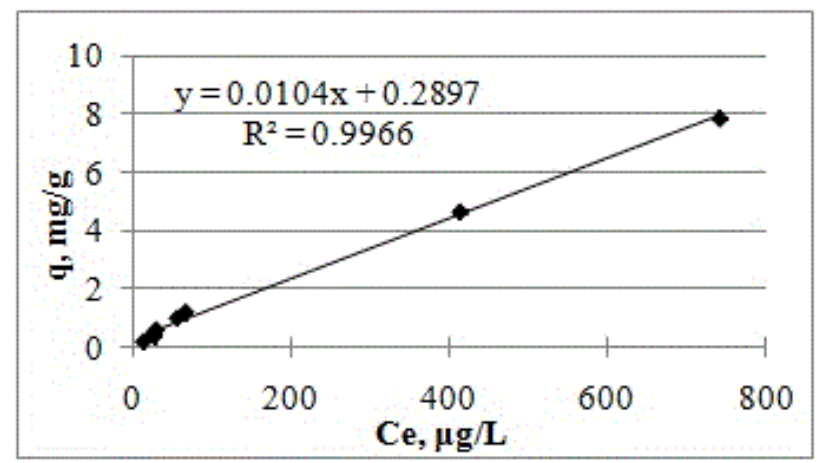

Figure 2. Linear isotherm for the uptake of TCE by $0.1 \mathrm{~g}$ ZSM- 5

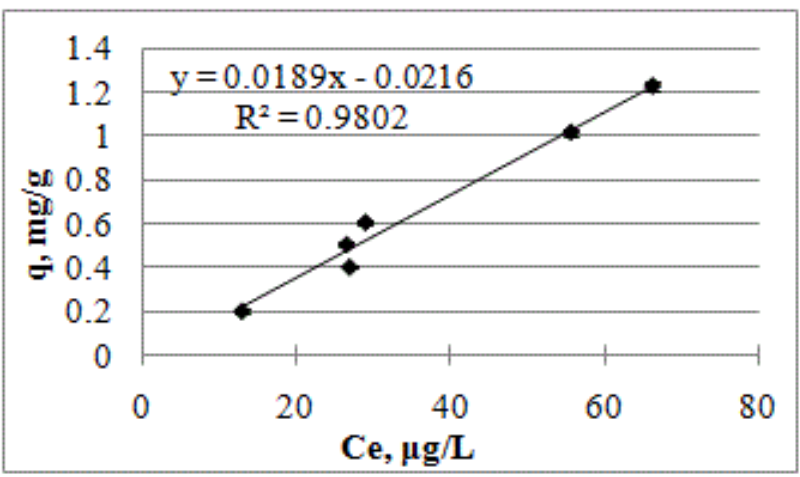

Figure 3. Low concentration linear isotherm of TCE on $0.1 \mathrm{~g}$ ZSM-5

$$
q_{e}=K C_{e}^{1 / n}
$$

Equation (1) can be linearized to (2).

$$
\log q_{e}=\log K+\frac{1}{n} \log C_{e}
$$

The Langmuir model is represented in (3), where $a$ and $b$ are characteristic constants which represent the maximum adsorption capacity and the net enthalpy of adsorption, respectively [11].

$$
q_{e}=\frac{a b C_{e}}{1+b C_{e}}
$$

Equation (3) can be linearized to (4).Equation (3) can be linearized multiple ways and this was taken into account 
during the analysis and (4) was the chosen as the most appropriate model [11].

$$
\frac{C_{e}}{q_{e}}=\frac{1}{a b}+\frac{C_{e}}{a}
$$

These models are shown in Figures 4 and 5.The Freundlich model resulted in a higher correlation $\left(\mathrm{R}^{2}=0.985\right)$ than the Langmuir model $\left(\mathrm{R}^{2}=0.8131\right)$. The linear isotherm had a higher correlation by only 0.0116 points (the enlarged linear isotherm which did not include the higher equilibrium concentrations had a lower correlation than the Freundlich model).If the experiments were conducted at higher concentrations (i.e.a higher sorption load), the data may deviate from the linear model and more closely follow a Freundlich model. However, the lower concentrations may be more representative of actual environmental contamination levels.

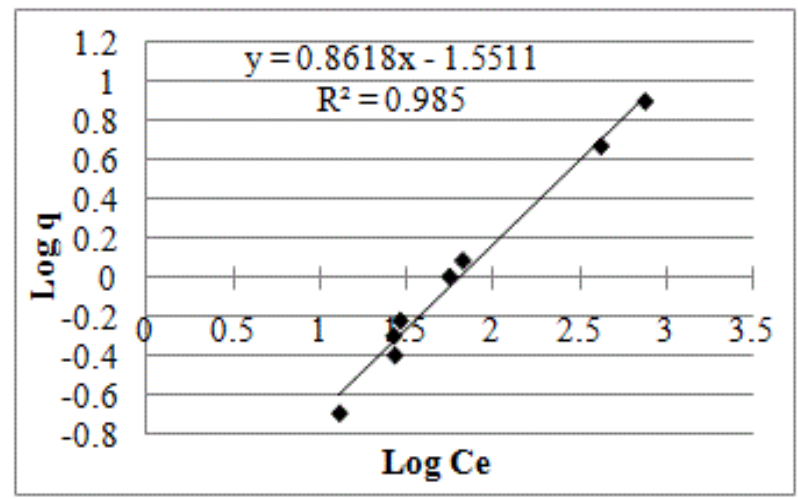

Figure 4. Freundlich model for TCE sorption to $0.1 \mathrm{~g}$ ZSM-5

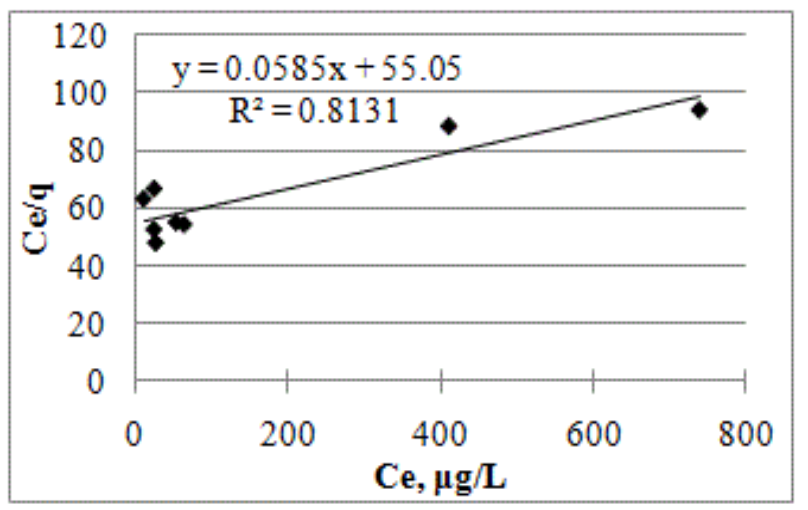

Figure 5. Linearized Langmuir model for TCE sorption to $0.1 \mathrm{~g}$ ZSM-5

Using the data in Figure 4 the characteristic Freundlich constants were determined $[13,14,15]$. The value for $1 / \mathrm{n}$, slope of the line was 0.8618 , and the value for $\mathrm{K}$, found from the y-intercept, was $0.028(\mathrm{mg} / \mathrm{g})^{*}(\mathrm{~L} / \mu \mathrm{g})^{1 / \mathrm{n}}$.

Giaya et al.[5] also modeled TCE sorption to silicalite (similar to ZSM-5, but with no aluminum) and found the data followed a Freundlich model.Their $1 / \mathrm{n}$ value was 0.702 and $\mathrm{K}$ was 0.43 using the same units as this study. The $\mathrm{K}$ value was about 15 times higher in the Giaya et al.[5] study. These values could differ due to differences in the zeolite composition. Giaya et al.[5] also used higher concentrations of TCE than in this study. Their equilibrium concentrations ranged from less than $500 \mu \mathrm{g} / \mathrm{L}$ to about $1,500 \mu \mathrm{g} / \mathrm{L}$ in the Giaya et al.[5] study versus equilibrium concentrations ranging from about 10 to $750 \mu \mathrm{g} / \mathrm{L}$ in this study, with the majority of the data at values less than $100 \mu \mathrm{g} / \mathrm{L}$.Li and Werth $[16,17]$ studied TCE uptake to H-ZSM-5 (similar to ZSM-5), but modeled the sorption of TCE using Langmuir models and modified Freundlich models to determine the sorption of TCE and of multiple organics in a single mixture. The data cannot be directly compared to this study, however the Li and Werth $[16,17]$ studies also found that TCE uptake followed a linear pattern when the $\log \mathrm{q}_{\mathrm{e}}$ and $\log$ $\mathrm{C}_{\mathrm{e}}$ are plotted, indicating that a Freundlich model was an acceptable representation of TCE sorption.

\subsection{Desorption}

After the supernatant was removed following equilibrium adsorption experiments, the vials containing the zeolite were filled with purified water.The vials were left to equilibrate for 24 hours on a shaker table and then were centrifuged. The supernatant was removed and the TCE that desorbed into the water was quantified using SPME and GC.It was found that if the original concentration was $500 \mu \mathrm{g} / \mathrm{L} \mathrm{TCE}$, there was less than $20 \mu \mathrm{g} / \mathrm{L}$ left in the water after sorption, and after desorption $11.5 \mu \mathrm{g} / \mathrm{L}$ desorbed from the zeolite. This experiment showed that significant amounts of TCE were reversibly adsorbed to the zeolite.Li and Werth [18] found that TCE desorbed from the zeolite H-ZSM-5 while studying slow desorption and that the time required for such desorption was less than 7 days (there are slight differences in the makeup of H-ZSM-5 and ZSM-5, however at this time the authors could not find desorption data on ZSM-5).

Modified $\mathrm{q}_{\mathrm{e}}$ values for the linear isotherm were determined to incorporate this potential desorption of TCE into the adsorption isotherm to create a modified isotherm incorporating concentration changes in the bulk solution. Thus, the modified $\mathrm{q}_{\mathrm{e}}$ is represented in (5), where $\mathrm{M}_{\text {desorbed }}$ is the mass of TCE that desorbed from the zeolite and $\mathrm{M}_{\text {zeolite }}$ is the mass of zeolite.

$$
q_{e}=\frac{M_{0}-M_{e}-M_{\text {desorbed }}}{M_{\text {zeolite }}}
$$

Figure 6 shows the original enlarged version of the linear isotherm with the modified $\mathrm{q}_{\mathrm{e}}$ isotherm plotted as well. As can be seen in the Figure, the data still follows the isotherm equation well, with the higher equilibrium concentrations showing the greatest deviation from the isotherm. The extent of desorption was low with the greatest standard deviation for a single desorption point being $0.017 \mathrm{mg} / \mathrm{g}$. The correlation was higher for the modified isotherm than for the adsorption isotherm, however both were acceptable values.This suggests that although the modified isotherm 
may be a more representative model, the original adsorption isotherm equation is still an acceptable model for the uptake of TCE by ZSM-5.

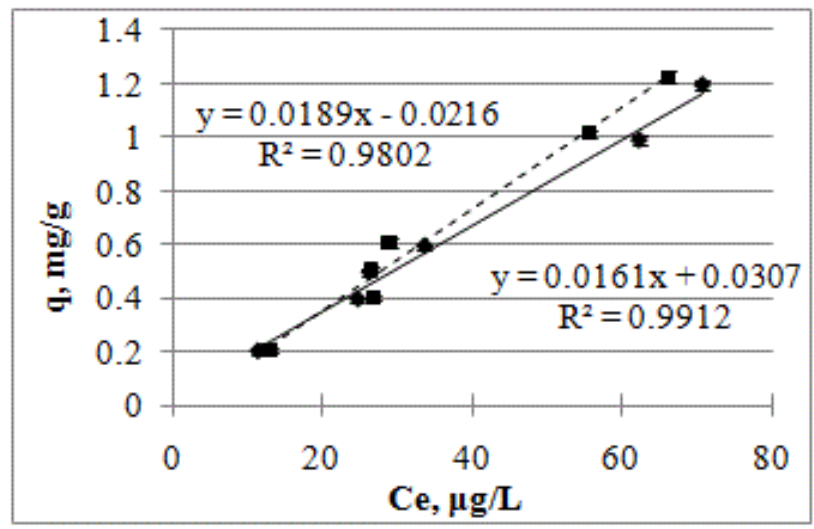

Figure 6. Linear adsorption and modified adsorption isotherms of TCE from ZSM-5

Wu et al.[19] studied the adsorption and desorption of the herbicide monosulfuron-ester to different soils. Wu et al.[19] also used a modified $\mathrm{q}_{\mathrm{e}}$ to incorporate desorption into the equilibrium adsorption model and found that the data followed the Freundlich model. The authors suggested that if the $1 / \mathrm{n}$ value of the adsorption isotherm was greater than the $1 / \mathrm{n}$ value for the desorption isotherm, the monosulfuron-ester was not easily removed from the soil.Using this principle, Freundlich isotherms were created for the adsorption and modified adsorption values for the lower concentration range as shown in the enlarged linear isotherm (Figure 6 ). The $1 / \mathrm{n}$ for adsorption was 1.08 , which was larger than that of the modified isotherm $(1 / \mathrm{n}=$ 0.96). The conclusion is that a small change was found in the model due to reversible adsorption, but adsorption was the principle mechanism.

The research reported here also provides a comparison to adsorption to granular activated carbon which has been shown to undergo desorption of contaminants as well [12,20,21].Zytner [12] found that TCE desorbed from granular activated carbon (GAC).In Zytner's study, $50 \mathrm{mg}$ of GAC was equilibrated with TCE concentrations ranging from 175 to $875 \mathrm{mg} / \mathrm{L}$ for 2 days, and then the GAC was allowed to equilibrate with deionized water for several days. Two separate Freundlich isotherms were created to model the adsorption and desorption as separate processes. Their study found that GAC retained the majority of the TCE.

A main goal of this project was to conduct adsorption and desorption experiments at environmentally relevant concentrations. Previous studies have shown that solid media released adsorbed organics when the organic concentration in the bulk solution was reduced in order to attain equilibrium [12,21].This can be seen during the desorption experiments where a lower concentration of TCE in the water resulted in desorption of some of the previously adsorbed TCE. Published research focuses on regenerating zeolites via oxidation of adsorbed organic contaminants $[22,23]$.It is important to note that desorption may occur and the contaminant would then need to be treated in the aqueous phase.

\section{Conclusions}

This study showed that ZSM-5 was able to rapidly adsorb the common groundwater contaminant TCE from the aqueous phase even from dilute solutions. Adsorption from dilute solutions followed linear and Freundlich isotherm models. Desorption of TCE from ZSM-5 occurred, however the linear isotherm still models the sorption of TCE from the aqueous phase well. As the adsorption and desorption isotherms were so close, it can be concluded that hysteresis was not observed to a great extent, and the adsorption process was essentially reversible. This suggests a physical adsorption process occurred between TCE and ZSM-5.

\section{REFERENCES}

[1] Michael A.Anderson, Removal of MTBE and Other Organic Contaminants from Water by Sorption to High Silica Zeolites, Environ.Sci.Technol., Vol.34, No.4, 725-727, 2000 .

[2] D.H.Olson, G.T.Kokotallo, S.L.Lawton, W.M.Meier, Crystal Structure and Structure-Related Properties of ZSM-5, J.Phys.Chem., Vol.85, 2238-2243, 1981.

[3] Andreas Jentys, Gerhard Warecka, Miroslav Derewinski, Johannes A.Lercher, Adsorption of Water on ZSM5 Zeolites, J.Phys.Chem., Vol.93, 4837-4843, 1989.

[4] Travis C.Bowen, Halil Kalipcilar, John L.Falconer, Richard D.Noble, Pervaporation of Organic/Water Mixtures Through B-ZSM-5 Zeolite Membranes on Monolith Supports, J.Membrane Sci., Vol.215, 235-247, 2003.

[5] Arjan Giaya, Robert W.Thompson, Raymond Denkewicz, Jr., Liquid and Vapor Phase Adsorption of Chlorinated Volatile Organic Compounds on Hydrophobic Molecular Sieves, Microporous Mesoporous Materials, Vol.40, 205-218, 2000.

[6] Wai Kit Chan, Justine Jouet, Samuel Heng, King Lun Yeung, Jean-Christophe Schrotter, Membrane Contactor/Separator for an Advanced Ozone Membrane Reactor for Treatment of Recalcitrant Organic Pollutants in Water, J.Solid State Chem., Vol.189, 96-100, 2012.

[7] Xiao-Liang Zhang, Mei-Hua Zhu, Rong-Fei Zhou, Xiang-Shu Chen, Hidetoshi Kita, Synthesis of a Silicalite Zeolite Membrane in Ultradilute Solution and Its Highly Selective Separation of Organic/Water Mixtures, Industr.Eng.Chem.Res., Vol.51, 11499-11508, 2012.

[8] Environmental Protection Agency (EPA).Sources, Emission and Exposure for Trichloroethylene (TCE) and Related Chemicals.EPA/600/R-00/099; National Center for Environmental Assessment-Washington Office (March, 2001). 
[9] Shujing Li, Yu-LunFang, Chris D.Romanczuk, Zhaohui Jin, Tielong Li, Michael S.Wong, Establishing the Trichloroethene Dechlorination Rates of Palladium-Based Catalysts and Iron-Based Reductants, Appl.Catal.B: Environ., Vol.125, 95-102, 2012.

[10] Ayse Erdem-Senatalar, John A.Bergendahl, Robert W.Thompson, Observations on Solid Phase Micro-Extraction for MTBE Analysis, Chemosphere., Vol.57, 523-527, 2004.

[11] Walter J.Weber Jr., Environmental Systems and Processes: Principles, Modeling, and Design, John Wily \& Sons, Inc., New York, 2001, pp 396-399.

[12] Richard G.Zytner, Adsorption-Desorption of Trichloroethylene in Granular Media, Water Air Soil Pollut., Vol.65, 245-255, 1992.

[13] American Water Works Association, Water Quality and Treatment: A Handbook of Community Water Supplies, Fourth Edition, Mc-Graw-Hill, Inc., New York, 1990, pp 782-803.

[14] Weilin Huang, Thomas M.Young, Mark A.Schlautman, Hong Yu, Walter J.Weber, Jr., A Distributed Reactivity Model for Sorption by Soils and Sediments.9.General Isotherm Nonlinearity and Applicability of the Dual Reactive Domain Model, Environ.Sci.Technol., Vol.31, No.6, 1703-1710, 1997.

[15] Ana M.Carmo, Lakhwinder S.Hundal, Michael L.Thompson, Sorption of Hydrophobic Organic Compounds by Soil Materials: Application of Unit Equivalent Freundlich Coefficients, Environ.Sci.Technol., Vol.34, No.20, 4363-4369, 2000.

[16] Jun Li, Charles J.Werth, Evaluating Competitive Sorption Mechanisms of Volatile Organic Compounds in Soils and Sediments Using Polymers and Zeolites, Environ.Sci.Technol., Vol.35, No.3, 568-574, 2001.

[17] Jun Li, Charles J.Werth, Modeling Sorption Isotherms of Volatile Organic Chemical Mixtures in Model and Natural Solids, Environ.Toxicol.Chem., Vol.21, No.7, 1377-1383, 2002.

[18] Jun Li, Charles J.Werth, Slow Desorption Mechanisms of Volatile Organic Chemical Mixtures in Soil and Sediment Micropores, Environ.Sci.Technol., Vol.38, No.2, 440-448, 2004.

[19] Chunxian Wu, Suzhi Zhang, Guo Nie, Zhongming Zhang, Jinjun Wang, Adsorption and Desorption of Herbicide Monosulfuron-ester in Chinese Soils, J.Environ.Sci., Vol.23, No.9, 1524-1532, 2011.

[20] Scott G.Huling, Robert G.Arnold, Raymond A.Sierka, Patrick K.Jones, Dennis D.Fine, Contaminant Adsorption and Oxidation via Fenton Reaction, J.Environ.Eng., Vol.126, No.7, 595-600, 2000.

[21] Priscilla C.To, Benito J.Marinas, Vernon L.Snoeyink, Wun Jern Ng, Effect of Pore-Blocking Background Compounds on the Kinetics of Trace Organic Contaminant Desorption from Activated Carbon, Environ.Sci.Technol., Vol.42, No.13, 4825-4830, 2008

[22] Duraiswami Divakar, Manuel Romero-Saez, Benat Pereda-Ayo, Asier Aranzabal, Jose A.Gonzalez-Marcos, Juan R.Gonzalez-Velasco, Catalytic Oxidation of
Trichloroethylene Over Fe-Zeolites, Catal.Today., Vol.176, 357-360, 2011.

[23] Prashant S.Chintawar, Howard L.Greene, Adsorption and Catalytic Destruction of Trichloroethylene in Hydrophobic Zeolites, Appl.Catal.B: Environ., Vol.14, 37-47, 1997. 